\title{
Atomic Structure of Defects in GaN:Mg grown with Ga polarity
}

Z. Liliental-Weber, T. Tomaszewicz, D. Zakharov, J. Jasinski, M.A. O’Keefe, S. Hautakangas ${ }^{\mathrm{a}}$, A. Laakso ${ }^{\mathrm{a}}$, and K. Saarinen ${ }^{\mathrm{a}}$

Lawrence Berkeley National Laboratory, Berkeley, CA 94720 m/s 62/203

${ }^{\mathrm{a}}$ Helsinki University of Technology. Espoo, Finland

\begin{abstract}
Electron microscope phase images, produced by direct reconstruction of the scattered electron wave from a focal series of high-resolution images, were used to determine the nature of defects formed in GaN:Mg crystals. We studied bulk crystals grown from dilute solutions of atomic nitrogen in liquid gallium at high pressure and thin films grown by the MOCVD method. All the crystals were grown with Ga-polarity. In both types of samples the majority of defects were three dimensional Mg-rich hexagonal pyramids with bases on the (0001) plane and six walls on $\{11 \underline{2} 3\}$ planes seen in cross-section as triangulars. Some other defects appear in crosssection as trapezoidal (rectangular) defects as a result of presence of truncated pyramids. Both type of defects have hollow centers. They are decorated by $\mathrm{Mg}$ on all six side walls and a base. The GaN which grows inside on the defect walls shows polarity inversion. It is shown that change of polarity starts from the defect tip and propagates to the base, and that the stacking sequence changes from $a b$ in the matrix to bc inside the defect. Exchange of the Ga sublattice with the $\mathrm{N}$ sublattice within the defect leads to $0.6 \pm 0.2 \AA$ displacement between Ga sublattices outside and inside the defects. It is proposed that lateral overgrowth of the cavities formed within the defect takes place to restore matrix polarity on the defect base.
\end{abstract}

\section{INTRODUCTION}

$\mathrm{GaN}$ can be easily grown with n-conductivity, at least up to a concentration of donors in the $10^{19} \mathrm{~cm}^{-3}$ range. On the other hand, p-doping remains still difficult and not well understood. The only efficient p-type dopant is $\mathrm{Mg}$, but the free hole concentration is limited to $2 \times 10^{18} \mathrm{~cm}^{-3}$ for $\mathrm{Mg}$ concentrations in the low $10^{19} \mathrm{~cm}^{-3}$ range. This could limit further development of $\mathrm{GaN}$ based devices. Further increase of the $\mathrm{Mg}$ concentration, up to $1 \times 10^{20} \mathrm{~cm}^{-3}$ leads to a decrease of the free hole concentration. This is commonly interpreted as auto-compensation due to increased formation of $\mathrm{N}$ vacancies or vacancy complexes with $\mathrm{Mg}$ [1]. Another characteristic feature is the occurrence of the so-called "blue band" in the photoluminescence spectra of highly Mgdoped GaN grown by MOVPE [2]. Transmission electron microscopy (TEM) studies show the formation of different types of $\mathrm{Mg}$-rich defects, both in bulk crystals grown from $\mathrm{Ga}$ excess under high N-pressure and in crystals grown by MOCVD [3-6]. The types of the defects strongly depend on crystal growth polarity (Fig. 1a). For bulk crystals grown with N-polarity, the planar defects (shown on Fig. 1a as lines) are distributed at equal distances (20 unit cells of GaN). These defects were described as inversion domains [6].

For growth with Ga-polarity (for both bulk and MOCVD grown crystals) a different type of defect has been found [4-6]. These defects are three-dimensional Mg-rich hexagonal pyramids with their base on the (0001) plane and six walls formed on $\{1123\}$ planes. The defects appear in [1120] and [1100] cross-section TEM micrographs as triangular and trapezoidal features with their base on the (0001) c-plane and six side facets, inclined at $43^{\circ}$ and $47^{\circ}$ to the base depending on the above observation directions, respectively (Fig. 1b). It appears that the dimension of these 
pyramids varies depending on growth method, but the angle between the base and their sides remain the same. In the bulk crystals the diameter of the base can be almost one order of magnitude larger (up to $1000 \AA$ ) than in thin foils grown by MOCVD $(50 \AA-150 \AA)$. In crosssection TEM micrographs a variety of defect sizes can be observed due to sectioning of the defect during TEM sample preparation (Fig. 2). The direction from the tip of the pyramid to its base is along the $\mathrm{Ga}$ to $\mathrm{N}$ matrix bond direction [2].

(a)
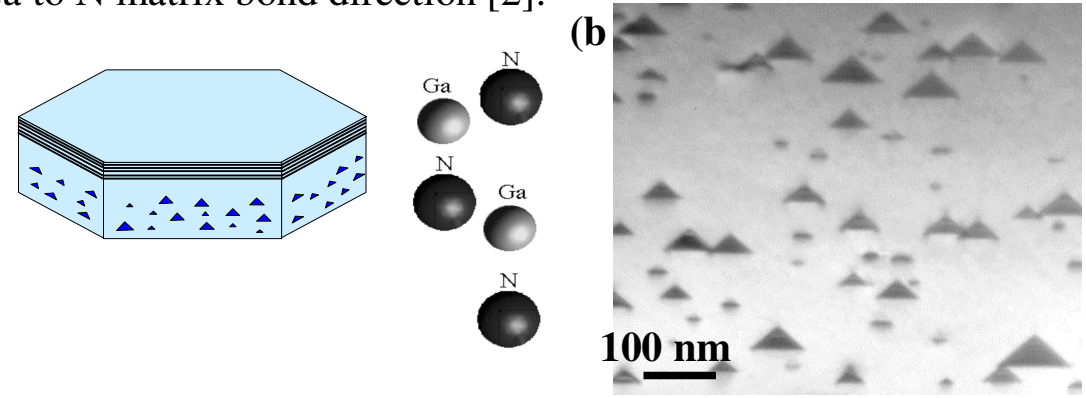

Fig. 1. (a) Schematic drawing shows defect distribution in a bulk GaN samples grown under high Ga pressure, together with atom arrangement indicating crystal polarity. (b) TEM micrograph shows defects in cross-section in [1120] projection formed in these crystals grown with Ga polarity. Note triangular and trapezoidal (almost rectangular) shapes.
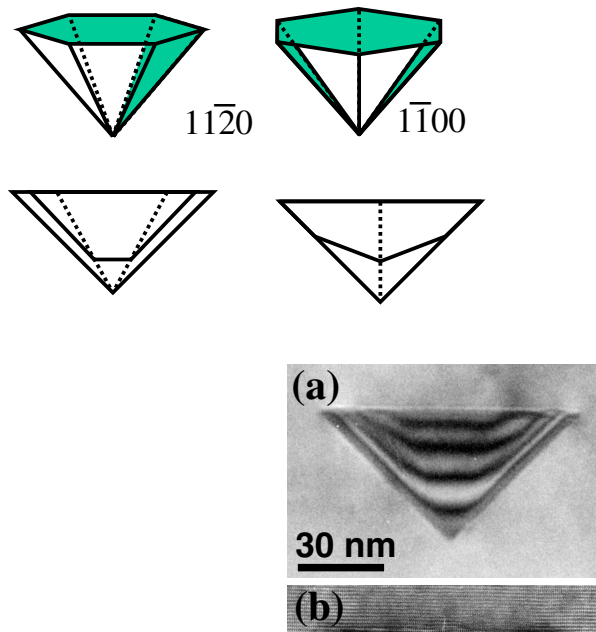

(b)

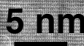

Fig. 2. Schematic drawings, in two crystallographic directions, of defects formed in GaN:Mg and their possible shapes due to sectioning during TEM sample preparation.

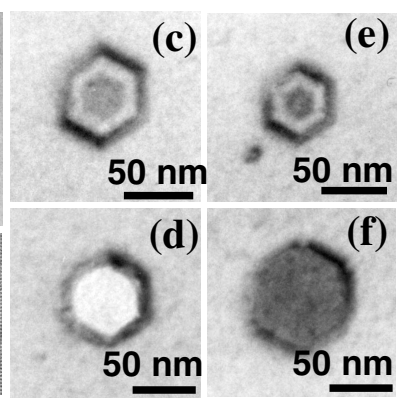

Fig. 3. TEM micrographs of two types of defects observed in cross-section samples: (a) a pyramid seen as triangular defect, (b) a truncated pyramid is seen as a trapezoid. (c-f) Same defects observed in plan-view samples. Their shapes indicate different sectioning in the planview TEM sample.

These triangular defects have often holes in their centers and are decorated by $\mathrm{Mg}$ on the defect walls [4-5]. An expansion of the interplanar spacing along the c-axis was measured on the defect base, suggesting Mg accumulation [6]. Other researchers have observed similar types of defects and described them as inversion domain inclusions [7-8]. The latest authors [8] confirmed our earlier observation [3] that the direction from the tip to the base of this defect is always from $\mathrm{Ga}$ to $\mathrm{N}$ and parallel to the c-axis. However, these authors observed formation of the pyramids in grains with $\mathrm{N}$ polarity $\left(180^{\circ}\right.$ rotated compared to the arrangement in grains with $\mathrm{Ga}$ 
polarity) instead of layer superlattice arrangement along the entire growth front, as was observed in bulk GaN grown with N-polarity [3]. This observation shows that, for MOCVD growth, rather small clusters are formed on the surface of the crystal grown with $\mathrm{N}$ polarity, that these further evolve into pyramids, and that formation of a particular defect in GaN is growth-method dependent.

For the trapezoidal features (Fig. 3b) a direction from the shorter to the longer base is also along the same $\mathrm{Ga}$ to $\mathrm{N}$ bond direction. These two defects cannot be distinguished in the plan-view configuration (Fig. 3c-f). They both appear as hexagons, therefore it is not clear if the trapezoidal features are the result of particular sectioning of the pyramids during TEM sample preparation or if these defects can start to grow up from an extended "patch" on the c-plane instead of growing from the tip (point).

\section{METHODS USED IN THESE STUDIES}

Pyramidal and trapezoidal defects formed in Ga-polarity bulk and MOCVD samples are characterized in this paper using several different electron microscopy methods. Methods include classical electron microscopy (CTEM) for determination of defect distribution and change of contrast for different diffraction conditions, convergent beam electron diffraction (CBED) for determination of defect polarity, and high resolution electron microscopy (HRTEM) to obtain high-resolution focal series for focal-series reconstruction of the electron exit-surface wave (ESW) leaving the specimen. Since the studied defects are small and faceted, the CBED method cannot be used routinely for polarity determination.

The phase of the complex electron wave at the specimen exit surface carries information about the specimen potential, projected in the direction of the incident electron beam. Cowley \& Iijima [9] showed that, without correction of spherical aberration by specialized hardware or focal-series reconstruction, imaging of the ESW phase is limited to the microscope resolution -about $1.6 \AA$ for mid-voltage TEMs. However, ESW phase information is available out to the microscope information limit [10]. This "extra" high-resolution information can be retrieved by focal-series reconstruction [11].

The reconstructed exit surface wave is a complex function of position, with phase peaks that correspond to atoms positions. The strength of each peak depends on the projected potential of the column of atoms underlying the peak. In turn, the potential depends on the number of atoms making up the column and their atomic number. With appropriate measurements, one can distinguish between different elements contributing to the generated phase and, for mono-atomic columns, also define the number of atoms in the column. Since heavier atoms produce more phase change, in GaN the phase change produced by a $\mathrm{Ga}$ atom column is always stronger than the phase change produced by a $\mathrm{N}$ column with the equivalent number of atoms (Fig. 4a,b). With increasing numbers of atoms in the columns (increasing thickness), the phase change moves to produce a counterclockwise Argand-plane trajectory of the complex ESW vector at the atom position. Since the change of phase for $\mathrm{Ga}$ is much faster than for $\mathrm{N}$ for the same number of atoms in the column, we can distinguish between $\mathrm{Ga}$ and $\mathrm{N}$ columns, determine crystal thickness and the crystal polarity. Color coding (not shown in this paper) was applied to present the complex exit surface wave map related to the vacuum wave as described earlier by Van Dyck \& Op De Beeck [12] and applied by Sinkler \& Marks [13]. In order to learn how the image contrast changes with sample thickness and stoichiometry, focal series were simulated using the MacTempas multislice programs [14]. 
(a)

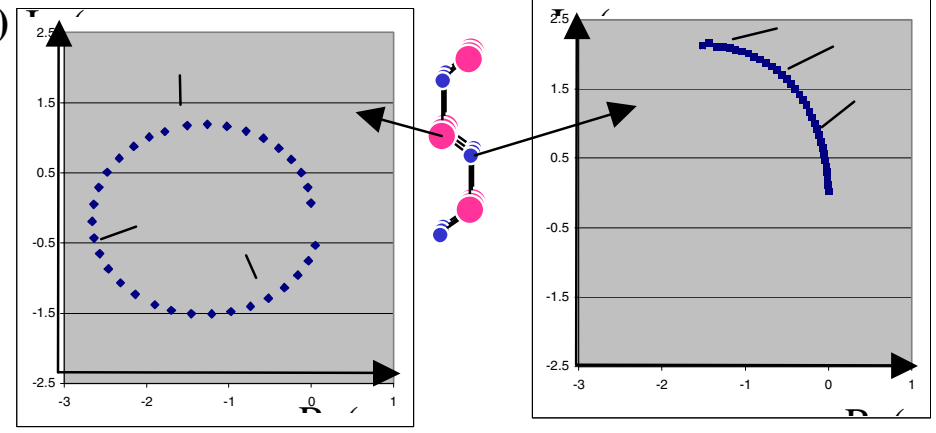

(b)

Fig. 4. Argand plot of a multislice-simulated GaN sample of the measured phase values for Ga (a) and $\mathrm{N}$ (b) for different number of atoms in the column.

The defects were also studied using Electron Energy Loss Spectroscopy (EELS) to determine their composition, as well as positron annihilation to determine the presence of vacancies and their conglomerations. Two types of samples have been studied: bulk samples grown by the high nitrogen pressure method from a solution of liquid gallium containing 0.1-0.5 at $\% \mathrm{Mg}$ [15] and samples grown by MOCVD described earlier [16] using $\mathrm{Cp}_{2} \mathrm{Mg}$ at $1030^{\circ} \mathrm{C}$.

\section{RESULTS}

\section{Bulk Samples}

Three different methods were applied to determine defect polarity. First, dark-field images under two beam condition for (0001) and (0001) diffraction vectors were obtained. If inversion operation is taking place then the areas of the crystal which fulfill this operation will reverse their contrast. This was observed for many of pyramids (Fig. 5).
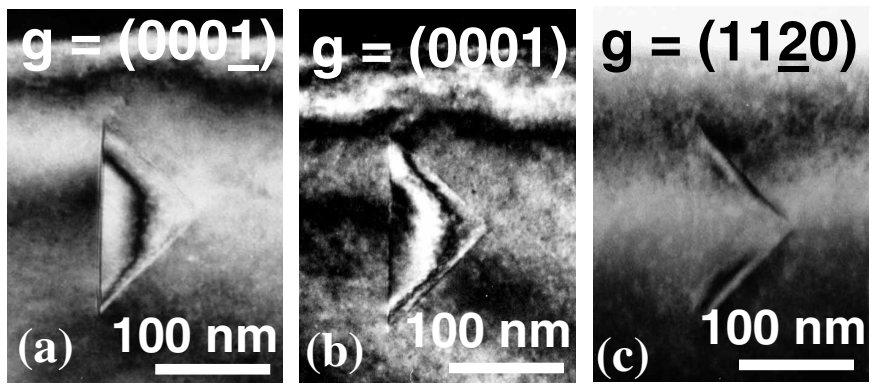

Fig. 5. Dark-field images taken with $g=0001$ (a) and (0001) (b) showing a reverse contrast. This is an indication that these defects are inversion domains. (c) Bright field image from the same defect taken with $g=1120$. Note a white fringe only slightly shifted when crossing the defect suggesting that TEM sample thickness is comparable in both areas (a matrix and a defect).

The black and white fringes reverse contrast when the (0001) diffraction vector is reversed (Figs. 5a,b) suggesting presence of inversion within the defect. However, when the defect is imaged with a perpendicular diffraction vector, only a small shift in the thickness fringe intersecting matrix and defect could be noticed, suggesting almost equal sample thickness in these two areas (Fig. 5c). When observed in cross-section projection, thickness within the defects very often appears to be smaller than in the surrounding matrix, with some defects containing an 
empty holes. This is true especially for trapezoidal defects (Fig. 6). When observed in plan view, two concentric hexagons can be observed within one defect; this would confirm that defect is empty inside [Figs. 3c-f].
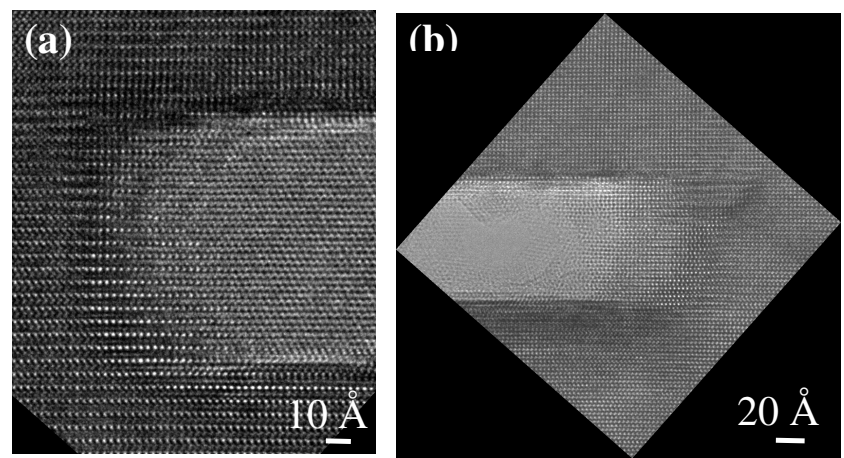

Fig. 6. High resolution image of two trapezoidal defects (only part of them are shown) showing different thickness within the defect and in the matrix (a) and a hole within the defect (b).

The second method which was applied was Convergent Beam electron Diffraction (CBED). More than 50 defects have been studied but only two of them gave interpretable patterns (Fig. 7). For this purpose the pyramid (marked by arrow in Fig. 7a) needs to be sectioned through the crystalline part of the defect which does not contain inclined walls. Whenever side walls are within the TEM sample than the scattering from these side walls makes the pattern very complicated. Figs. $7 \mathrm{~b}$ and $7 \mathrm{c}$ were taken from a pyramid and a matrix, respectively. One can notice reverse change of the intensity within the same disc (0002) and (0002) for the defect and the matrix. The calculated patterns indicate polarity reversal and smaller thickness within the pyramid.

The third method was high resolution electron microscopy for exit wave reconstruction. Much better statistics could be obtained in this case. For many defects it was possible to obtain a cross-section TEM sample with a pyramid and its tip at the same sample thickness. It was determined that majority of the pyramids had empty holes inside and only part of the defect adjacent to the pyramid side walls and a base had a crystalline layer.

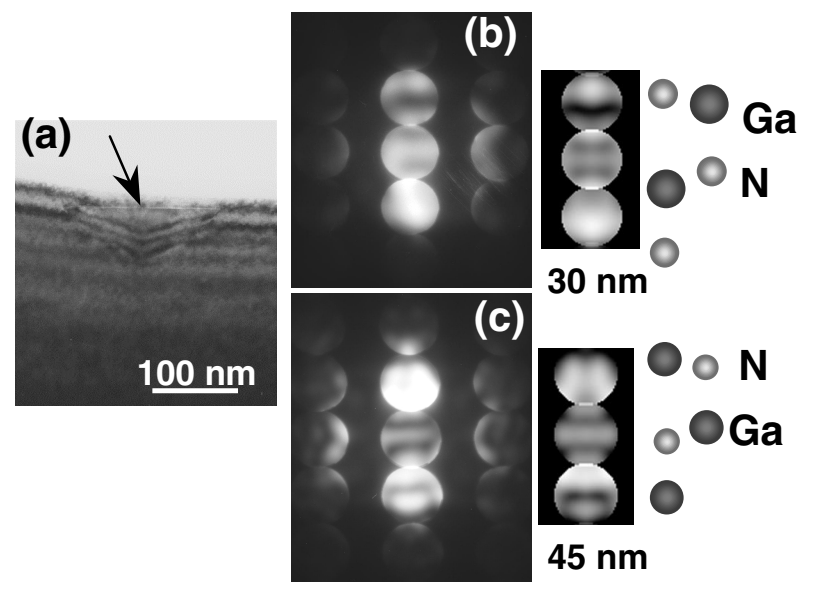

Fig. 7. A bright field of the pyramid (marked by arrow) in a cross-section sample (a); (b) and (c) CBED patters from the pyramid and the matrix, respectively, together with calculations for specific sample thickness and indicated atom arrangement $(\mathrm{N}-$ and $\mathrm{Ga}-$ polarity for the pyramid and the matrix, respectively).

Reconstructed exit wave phase from the area close to the pyramid tip (Fig. 8) showed blobs with two distinct intensity peaks, identified as due to Ga and $\mathrm{N}$ atomic columns. Using Argand plots, we confirmed that the phase of atoms described as Ga gave the highest peak, 
followed by a smaller phase peak described as $\mathrm{N}$, as expected from earlier theoretical calculations. This identification of the atomic positions of $\mathrm{Ga}$ and $\mathrm{N}$ from the experimental reconstructed exit wave confirmed inversion of polarity within the pyramid compared to the matrix. In addition, it showed ab stacking in the matrix changes to bc stacking within the pyramid (Figs. 8b-d). This stacking arrangement holds through the entire pyramid and changes back to the ab stacking order above the pyramid base.

Since the growth direction of the layer is from a pyramid tip to the base it is also expected that polarity change follows the growth direction. Pyramid start to form from the $\mathrm{Ga} / \mathrm{Mg}$ clusters which are often observed at the pyramid tip (will be shown on MOCVD grown films Fig. 11a).
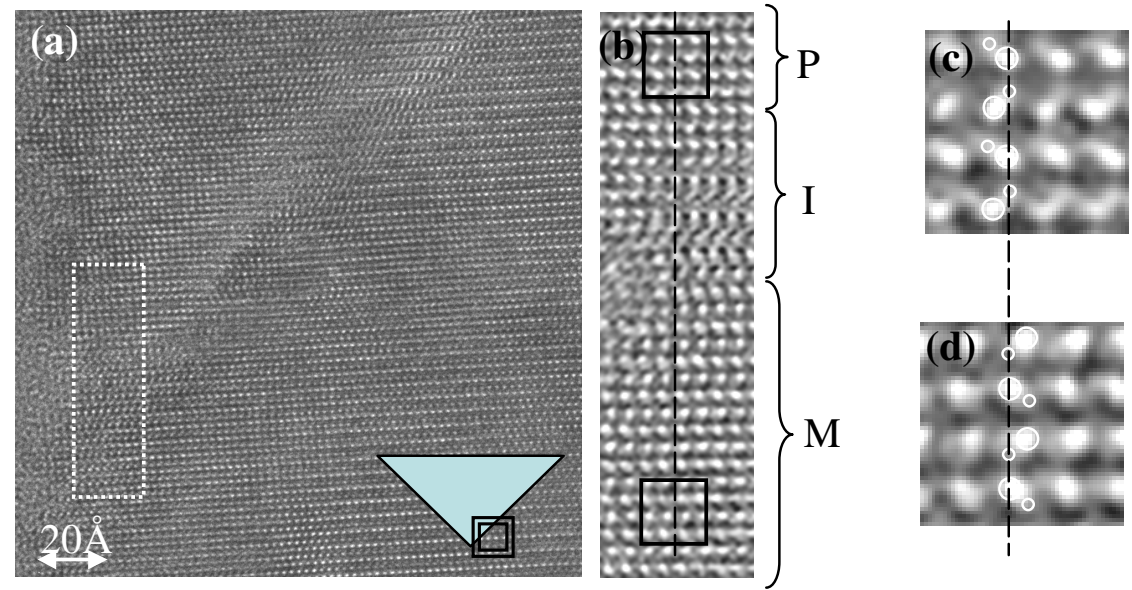

Fig. 8. Cross-section image from the part of pyramid close to the pyramid tip, schematically shown as inset in (a), (b) reconstructed exit wave phase from the side interface: P-inside the pyramids, I-the side interface area, M-the matrix below the side interface, (c) and (d) magnified reconstructed exit wave phase from the marked areas shown in (b) from the pyramid and the matrix, respectively, in high magnification with indicated atom position. Note stacking change within the defect (bc) compared to the matrix (ab).

It was also noticed that for the planes close to the pyramid base it was difficult to determine $\mathrm{N}$ atomic column positions. Sometime it was impossible to determine $\mathrm{N}$ atomic columns on one c-plane close to the pyramid base but sometimes on 2 or 3 parallel planes. This would mean that $\mathrm{N}$ positions were occupied by different type of atoms. Since these atomic columns had phases smaller than the surrounding Ga columns above the base, they could be due to the presence of $\mathrm{Mg}$ in this part of defect. Different sample thickness can be ruled out, since this phase change is limited only to a few monolayers at the base, and must be primarily due to $\mathrm{Mg}$ presence. This is consistent with a larger separation between c-planes measured across the base. Increase in separation starts at $0.2 \AA$ above the pyramid and can grow, in some cases, up to $0.6 \AA$ at the pyramid base. Expansion of the c-planes would be consistent with the decoration of several planes close to the base by $\mathrm{Mg}$ or $\mathrm{MgO}$.

Analysis of the reconstructed exit wave phase image from the pyramid side (Fig. 9) indicates a shift of $\mathrm{Ga}$ atomic column positions from the matrix to the $\mathrm{N}$ position within the pyramid. In this way a $0.6 \AA \pm 0.2 \AA$ displacement can be measured on the pyramid side between Ga positions in the matrix and within the pyramid.

Studies of polarity in trapezoidal defects occasionally showed change of polarity, but in the majority of cases it was difficult to determine due to the presence of large tilts on the defect sides or of large holes within the defects. The density of the trapezoidal defects was always 
larger than the triangular defects, and the holes inside these defects were always larger. This was true for the bulk crystals and also for these grown by MOCVD.

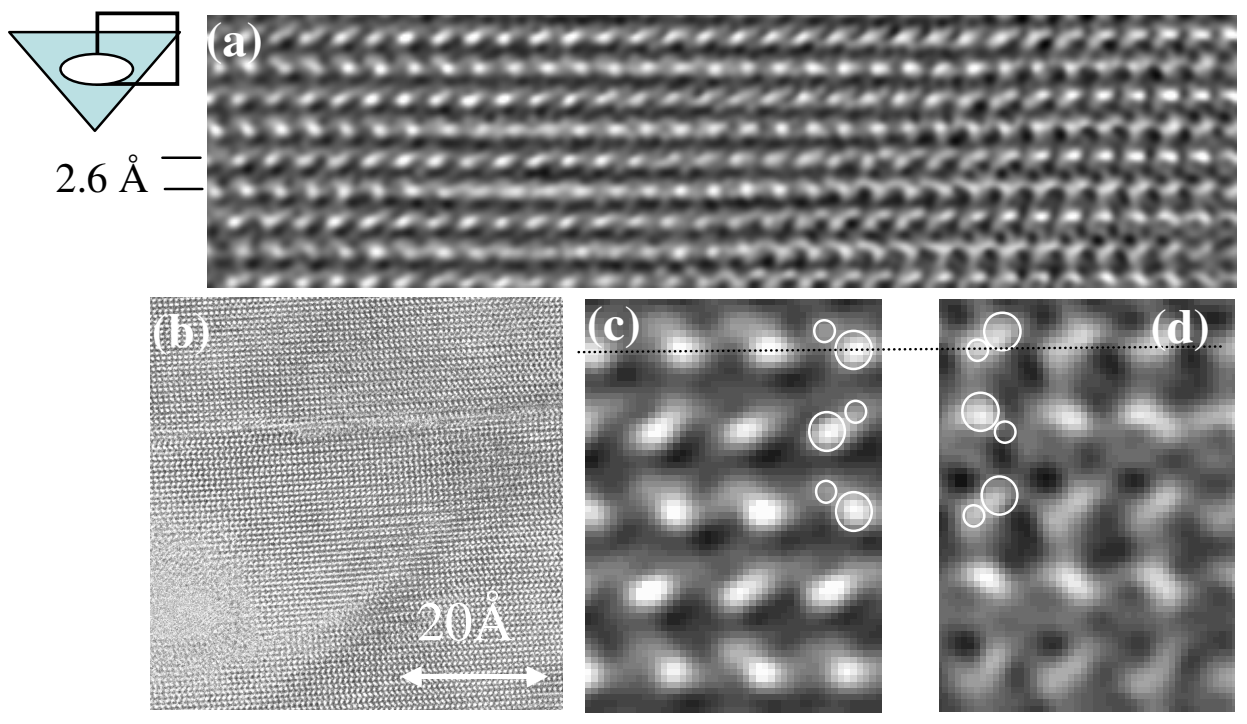

Fig. 9. (a) Reconstructed exit wave phase image from the pyramid side (a higher magnification from the box marked on the schematic in the upper left corner; (b) reconstructed exit wave phase image from the larger area of the pyramid (schematically showed on the inset). Note hole present in the lower left corner; (c) and (d) very high magnification of the reconstructed wave phase image from the pyramid and from the matrix close to the pyramid side, respectively, aligned along one c-plane, together with marked atomic columns. Note that Ga positions in the matrix exchange positions with the $\mathrm{N}$ atomic columns within the defect leading to $0.6 \AA$ displacement between Ga sublattices within the pyramid and in the matrix.

Energy loss spectra were taken from both triangular and trapezoidal defects. For the triangular defect, prepared from the areas closer to the center part of the pyramid (but not touching the hole) there were no differences for the $\mathrm{NK}_{\alpha}$ peak position and its shape outside the defect and within the defect (Fig. 10a). This result confirms our earlier observations [4-6] that decoration by $\mathrm{Mg}$ is only present on the defect walls and that inside the pyramid no new phase is formed. Our studies do not find the $\mathrm{Mg}_{2} \mathrm{~N}_{3}$ phase as reported earlier [17].

The $\mathrm{NK}_{\alpha}$ peak from the matrix area contains three small maxima (marked $\mathrm{A}, \mathrm{B}$, and $\mathrm{C}$ on Fig. 10a) with almost equal heights (B has slightly higher intensity). These peaks did not change when defect areas were scanned (dots on the inset show beam positions for the spectra shown in (a)). However, a large change in the $\mathrm{NK}_{\alpha}$ peak was observed (Fig. 10b) when the defect side wall was within the TEM thin foil specimen (for both triangular and trapezoidal defects). The intensity of the peak A of the $\mathrm{NK}_{\alpha}$ increased substantially. Such a peak change is usually connected with the structural change, most probably due to formation of a cubic phase. When similar spectra were taken from the trapezoidal defect containing a hole, an increase of the intensity of the A peak was observed whenever an electron beam was placed directly on the lower base (see third spectrum from the bottom on Fig. 10c). The intensity of the peak disappeared when beam was placed within the hole, then returned to the same arrangement as observed earlier above a hole. Simultaneously an oxygen edge was observed. A substantial 
intensity of oxygen was observed when beam intersected the lower and the higher base of this defect (Fig. 10d). This would mean that oxygen is present on the defect walls and might be attached to $\mathrm{Mg}$, possibly forming $\mathrm{MgO}$. This compound could be responsible for the increase of A maxima shown on Fig. 10b.
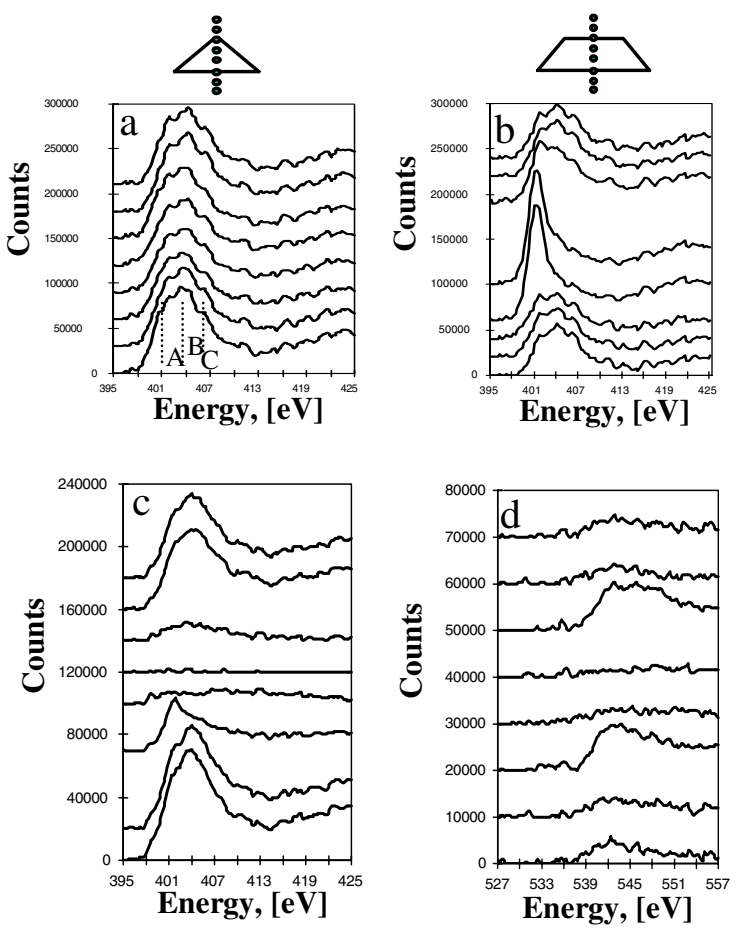

Fig. 10. (a) Electron Energy Loss Spectra (EELS), the $\mathrm{NK}_{\alpha}$ edge, from the center of the triangular defect; (b) The $\mathrm{NK}_{\alpha}$ edge from the side of the trapezoidal defect. Note an increase of intensity of A maximum. (c) The $\mathrm{NK}_{\alpha}$ edge from the center of the trapezoidal defect with a hole inside. Note slight increase of the intensity of the A maximum when an electron beam intersects the lower base (third spectrum from the bottom) and flattening of the peaks when the electron beam hits the hole. (d) The $\mathrm{OK}_{\alpha}$ edge from the same defect taken simultaneously with the spectra shown in (c). Note an increase of oxygen peak when the electron beam intersect the lower and the upper base of the defect. Schematics at the top show defect shapes and positioning of the electron beam for spectra accumulation.

\section{Defects in GaN:Mg grown by MOCVD}

Similar types of defects were observed in crystals grown by low-pressure metal-organic chemical-vapor deposition (MOCVD). The studied crystals had comparable Mg concentrations of about $6 \times 10^{19} \mathrm{~cm}^{-3}$. Mg-rich defects did not appear immediately when the $\mathrm{Mg}$ precursor $\left(\mathrm{Cp}_{2} \mathrm{Mg}\right)$ was switched on. First a layer free of defects was formed, followed by formation of the triangular and trapezoidal defects. Defect distribution was not uniform; there were areas where density of these defects was as high as $10^{10} \mathrm{~cm}^{-2}$ adjacent to areas where defect density dropped by one or two orders of magnitude [5]. When measured on the defect base, these triangular defects, with diameters in the range of $30-150 \AA$, were much smaller in comparison with those in bulk crystals. Enhanced contrast suggesting decoration on defect walls was often present on the observed defects (Fig. 11a). In $11 \underline{20}$ and 1100 projections, these defects always showed a different pattern than the surrounding matrix, consistent with a smaller thickness as calculated using MacTempas program [14]. As in the case of bulk crystals, clusters of $\mathrm{Ga} / \mathrm{Mg}$ atoms were often found near their tips (Fig. 11a), showing that the mechanism of their formation must be the same in both types of crystals. The shift of the c- lattice planes, observed on the defect side (Fig, $11 b)$ at Scherzer defocus, shows not only the displacement between Ga planes in the matrix and the defect, but is also related to the difference in thickness between these two areas. Reconstruction of the ESW from series of TEM high-resolution micrographs was used to determine the shift between Ga atomic column positions outside and inside the defect. 

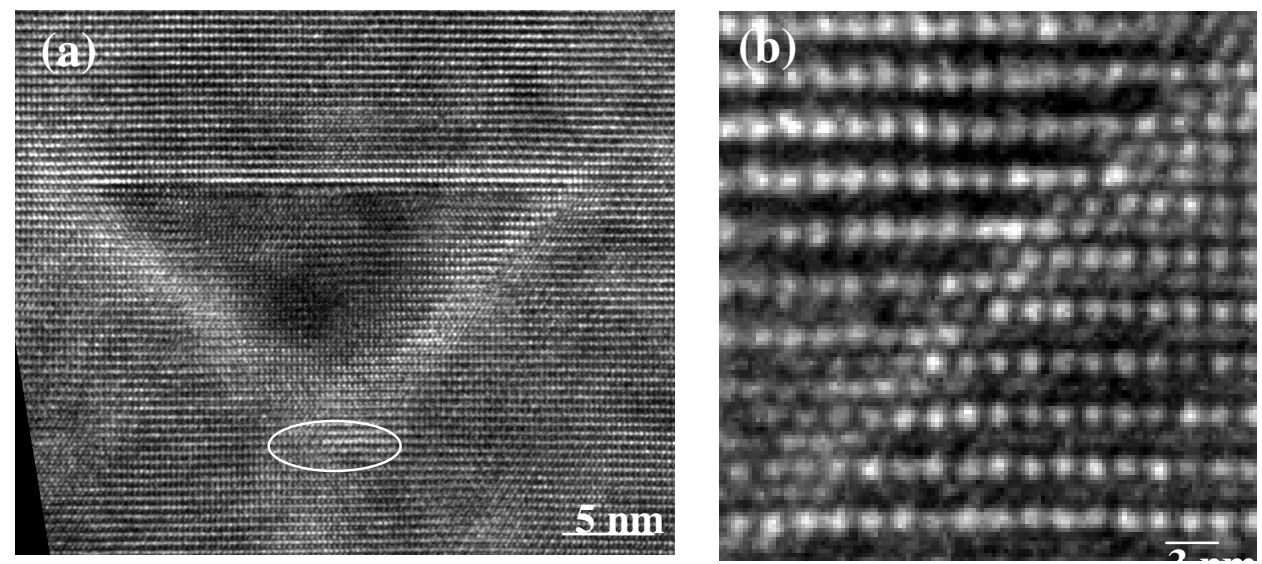

Fig. 11. (a) High resolution micrograph in 1120 projection of a pyramidal defect in the MOCVD sample. Note contrast enhancement on the side walls and the defect base and a $(\mathrm{Ga} / \mathrm{Mg})$ cluster formed close to the defect tip (marked). (b) Contrast enhancement on different c-planes in the matrix and the defect is mainly due to the different sample thickness in these two areas.
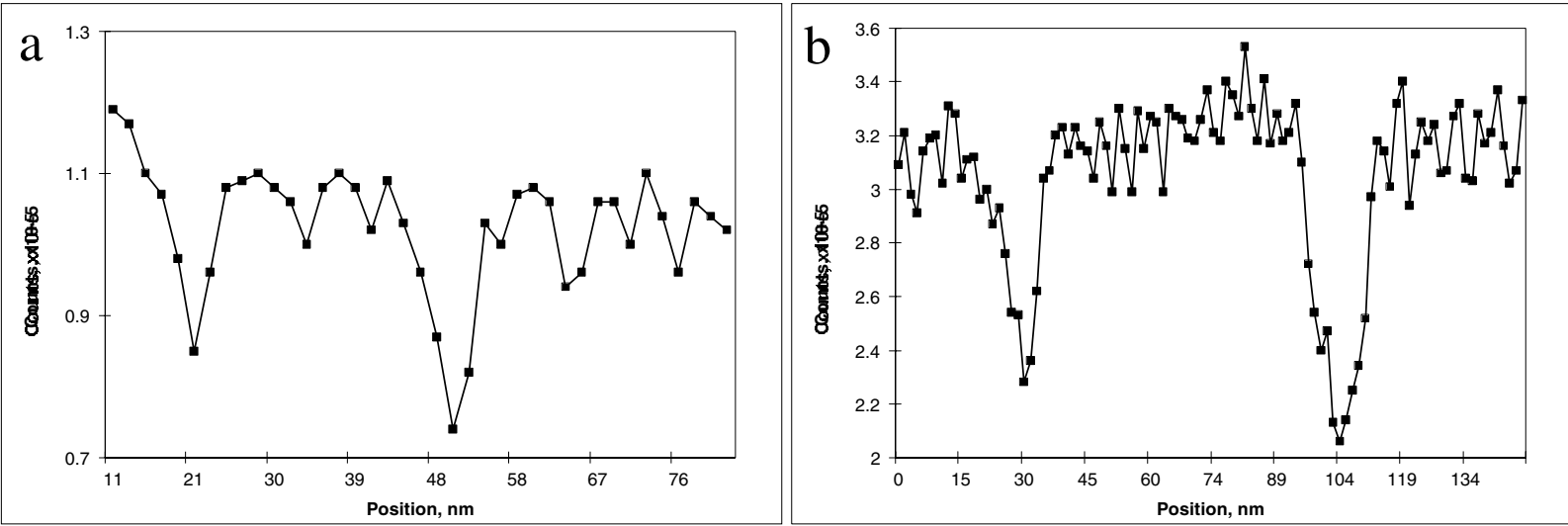

Fig. 12. EELS spectra from the GaN:Mg sample: (a) intensity of $\mathrm{GaK}_{\alpha}$ and (b) $\mathrm{NK}_{\alpha}$ lines as a function of beam position. Drops in signal strength occur when the electron beam crosses a pyramidal defect, confirming that defect areas are much thinner than the bulk.

Because small pyramids are often covered by matrix material, it was very difficult to obtain a good set of images useful for ESW reconstruction. Our calculations showed that coverage of the defect by only five monolayers of matrix would not allow proper reconstruction since both $\mathrm{Ga}$ atoms from the defect and the matrix contribute to the image. However, some reconstructions were successful, especially those from 1100 projection, where the side walls of pyramidal defects are edge on. In this orientation, we could identify Ga atomic columns on cplanes outside the pyramid and in the matrix and measure $0.6 \AA$ displacement. This is exactly the same displacement measured in bulk crystals. Since this displacement on the side wall, and the angle between the base and a side wall, remain the same as observed in the bulk GaN:Mg crystals, it is believed that defects formed in both types of crystals are similar. Based on images 
taken at Scherzer defocus distances between c-planes across the defect base were estimated to be larger, comparable to those measured in bulk crystals (with $\Delta \mathrm{c}$ up to $0.6 \AA$ ).

Additional information provided by exit wave reconstruction is that areas with defects are much thinner. This result is confirmed by EELS studies; when the electron beam passes defect areas, the intensity of $\mathrm{GaK}_{\alpha}$ and $\mathrm{NK}_{\alpha}$ lines decreased by more then $30 \%$ (Fig. 12) confirming the thickness decrease in defect areas.

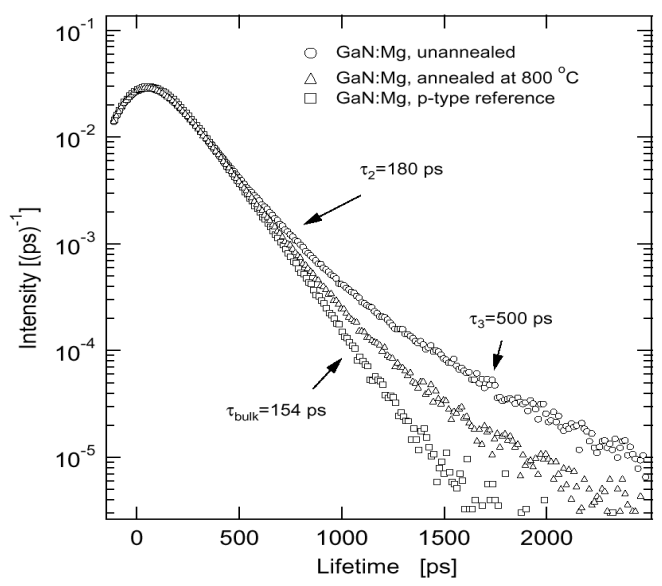

Fig. 13. Positron lifetime spectra measured in $\mathrm{Mg}$ doped GaN grown by MOCVD show three distinct curves: $\tau_{1}=154 \mathrm{sec}, \tau_{2}=180$ sec, and $\tau_{3}=500 \mathrm{sec}$ The pulsed positron beam energy was $16 \mathrm{keV}$ and measuring temperature $540 \mathrm{~K}$.

The MOCVD samples were studied using positron annihilation. The positron lifetime spectra consist of exponential decay components $\tau_{\mathrm{i}}$ with an intensity $\mathrm{I}_{\mathrm{i}}$. By decomposing the spectra, positron lifetimes and annihilation fractions at free and trapped positron states can be resolved. In Mg doped samples grown by MOCVD there is a strong evidence of vacancy defects (Fig. 13). Three lifetime components can be distinguished: $\tau_{1}=154 \mathrm{sec}, \tau_{2}=180 \mathrm{sec}$, and $\tau_{3}=500$ sec. The longest lifetime is typical for large open volume defects where at least 10 atoms are missing. This longest lifetime observed in these samples and in the GaN:Mg samples studied earlier [18] would be consistent with the cavities within pyramidal and truncated defects that were observed in our TEM studies. Furthermore, the depth distribution of the open volume detected by positron annihilation is similar to that observed for the pyramidal and truncated defects observed by TEM.

\section{MODELS AND RESULTS DISCUSSION}

Several models for inversion domains in GaN:Mg have been proposed. Our two models [4], proposed for GaN:Mg grown with $\mathrm{N}$-polarity and applied for flat interfaces between N-polar and Ga-polar crystals, introduced a shift of $[1 \underline{100}] / 3+\mathrm{c} / 2$. This shift is also consistent with our present observation of pyramidal defects observed not only on the pyramid base, as discussed by Northrup [19], but also on the pyramid side. The abcab model described by Northrup [19] has the lowest energy but it is not consistent with our experimental data since it considers only one layer rich in $\mathrm{Mg}$ on the $\mathrm{c}$ position. The $\mathrm{Mg}$ on this position would need to be 6-fold coordinated. According to calculations by Northrup [19] our model would be energetically less favorable, but $\mathrm{Mg}$ could be 4-fold coordinated surrounded by 6-coordinated $\mathrm{N}$ and it would include change of stacking sequence from $a b$ to $b c$ and a shift on the pyramid side of $0.6 \pm 0.2 \AA$ between Ga sublattices in the matrix and the defect. Romano [20] suggested that the Ga sublattice continues across the pyramid side and Leroux [21] considered that the $\mathrm{N}$ sublattice continues across the same boundary, as similarly proposed by Northrup [19]. We believe that only our method gives 
enough resolution to see particular sublattices which was not presented by any other author up to now.

Both our earlier [3-6] and present observations show very sharp base interfaces and well defined angles between the pyramid side and its base. It is difficult to imagine that a change of polarity due to $\mathrm{Mg}$ presence could start so abruptly at this interface and go down to the pyramid tip after growth. Rather, we believe that the polarity change initiates at $\mathrm{Ga} / \mathrm{Mg}$ clusters, which are often observed in both bulk and MOCVD grown crystals. Considering the $43^{\circ}$ and $47^{\circ}$ angles between pyramid sides observed in $11 \underline{20}$ and 1100 respectively, one can notice that this plane is equally occupied by $\mathrm{Ga}$ and $\mathrm{N}$, therefore $\mathrm{Mg}$ can be attracted by both these atoms. Ga can be substituted by $\mathrm{Mg}$, as expected for Ga polarity, and $\mathrm{N}$ can be attracted by $\mathrm{Mg}$ since $\mathrm{Mg}-\mathrm{N}$ compounds can be easily formed. In this way, two atomic layers rich in $\mathrm{Mg}$ can be formed on the defect walls. It can also happen that some $\mathrm{N}$ vacancies are formed on the side interface, or some of them are exchanged by oxygen consistent with our EELS results. This substitution would be consistent with charge neutrality at these side interfaces, but precise calculations would be needed to determine the energetically-favorable interface.

Once the stacking sequence and polarity change at the pyramid side walls, this sequence will propagate to the base, changing again to the matrix arrangement above the base. We believe that decoration by $\mathrm{Mg}$ on the defect sides is quite fast, however the growth of GaN with the opposite polarity $(\mathrm{N})$ would be much slower leading to meniscus formation. A minute lack of $\mathrm{Mg}$ on the defect walls would lead to fast lateral overgrowth along c-planes, since this growth direction is much faster than along c-direction. Since $\mathrm{Mg}$ always floats on the surface, some rearrangement of $\mathrm{Mg}, \mathrm{Ga}$ and $\mathrm{N}$ would take place below the base (inside the pyramid), therefore it is not surprising to observe one or more c-planes rich in $\mathrm{Mg}$ close to the defect base. This lateral overgrowth would ensure an abrupt interface on the defect base and a return to the matrix polarity.

\section{SUMMARY}

Transmission electron microscopy and reconstructed exit wave phase imaging were applied to study defects formed in bulk and MOCVD GaN:Mg crystals. Two types of defects have been found: hexagonal pyramids with 6 inclined walls and a base formed on c-plane, and truncated pyramids. It is believed that the latest defects are most probably formed from the larger clusters accumulating on c-planes, but it is also possible that their appearance in this form is only a result of TEM sample preparation. These defects have a hollow core, but their walls are covered by a substantial thickness of GaN grown with opposite polarity. These imaging results were confirmed by EELS and positron annihilation studies. Our EELS studies also show the presence of oxygen and the formation of a different crystal structure on the defect walls, but they do not confirm presence on $\mathrm{Mg}_{3} \mathrm{~N}_{2}$ as suggested earlier. Exchange of a Ga sublattice with a $\mathrm{N}$ sublattice within the defect leads to $0.6 \pm 0.2 \AA$ displacement between the Ga sublattices outside and inside the defects. It is shown that change of polarity starts from the defect tip and propagates to the base. Lateral overgrowth of the cavities formed within the defect is proposed to take place to ensure a return to matrix polarity on the defect base. 
This work was supported by the U.S. Department of Energy under contract DE-AC0376SF00098. Use of the OAM facility at the National Center for Electron Microscopy at the LBNL is greatly appreciated.

\section{REFERENCES:}

1. U. Kaufman, P. Schlotter, H. Obloch, K. Kohler, M. Maier, Phys. Rev. B62, 10867 (2002).

2. S. Nakamura and G. Fasol, "The blue laser diode" (Springer-Verlag, Berlin (1997).

3. Z.Liliental-Weber, M. Benamara, J. Washburn, et al, Phys. Rev. Letter. 82, 2370 (1999).

4. Z. Liliental-Weber, M. Benamara, W. Swider, J. Washburn, I. Grzegory, S. Porowski, R.D. Dupuis, and C.J. Eiting, Physica B 273-274, 124 (1999).

5. Z. Liliental-Weber, M. Benamara, J. Washburn, I. Grzegory, S. Porowski, D.J.H. Lambert, C.J. Eiting, and R.D. Dupuis, Appl. Phys. Lett. 75, 4159 (1999).

6. Z. Liliental-Weber, J. Jasinski, M. Benamara, I. Grzegory, S. Porowski, D.J.H. Lambert, C.J. Eiting, and R.D. Dupuis, Phys. Stat. Sol. (b) 228, No. 2, 345 (2001).

7. P. Vennegues, M. Benaissa, S. Dalmaso, M. Leroux, E. Feltin, P. De Mierry, B. Beaumont, B. Damilano, N. Grandjean, and P. Gibbar, Mat. Science and Engin. B93 , 224 (2002).

8. R. Kroger, S. Figge, T. Bottcher, P.L. Ryder, and D. Hommel, Mat. Res. Symp. Proc. 693, 615 (2002).

9. J.M. Cowley and S. Iijima, Z. Naturforsch., 27a, 445 (1972).

10. M.A. O'Keefe, Ultramicroscopy 47, 282 (1992).

11. A. Thust, W.M.J. Coene, M. Op De Beeck, and D. Van Dyck, Ultramicroscopy 64, 211 (1996).

12. D. Van Dyck and M. Op De Beeck, Ultramicroscopy 64, 99 (1996).

13. W. Sinkler and L. D. Marks Ultramicroscopy 75, 251 (1999).

14. M.A. O'Keefe and R. Kilaas, Scanning Microscopy suppl. 2, 225 (1988

15. S. Porowski, M. Bockowski, B. Lucznik, et al, Acta Phys. Pol. A92, 958 (1997).

16. C.J. Eiting, P.A. Grudkowski, J.S. Park, et al J. Electr. Soc. 144, L219 (1997).

17. D.P. Bour, H.F. Chung, W. Gotz, L. Romano et al, Mater. Res. Soc. Symp. 449, 509 (1997).

18. S. Hautaakangas, J. Oila, M. Alatalo, K. Saarinen, L. Liszkay, D. Seghier, and H.P. Gislason, Phys. Rev. Lett. 90, No. 13, 137402, (2003).

19. J.E. Northrup, Appl. Phys. Lett. 82, 2278 (2003).

20. L.T. Romano, J.E. Northrup, A.J. Ptak, and T.H. Myears, Appl. Phys. Lett. 77, 2479 (2000).

21. M. Leroux, P. Vennegues, S. Dalmasso, M. Benaissa, et al. Phys. Stat. Sol. 192, 394 (2002). 